\title{
Detection of hydrogen by electron Rutherford backscattering
}

\author{
Maarten Vos \\ Atomic and Molecular Physics Laboratories, Research School of Physical Sciences and Engineering, The Australian National University, \\ Canberra, ACT 0200, Australia
}

Received 12 July 2001; received in revised form 13 December 2001

\begin{abstract}
A novel method for detection of hydrogen by an electron beam in extremely thin samples is described. Elastically scattered electrons impinging with $20-30 \mathrm{keV}$ on a thin formvar film were detected at a scattering angle near $45^{\circ}$. In these large momentum transfer elastic collisions a clear separation of the signal of hydrogen and heavier elements was found. By changing the momentum transfer we can verify that the hydrogen signal is not due to inelastic energy loss contributions. The width of the hydrogen elastic peak is much larger than the elastic peaks due to heavy elements (carbon and oxygen). The ratio of the hydrogen elastic peak and the main elastic peak is smaller than expected by $30-50 \%$ depending on the energy of the impinging electron. This could be due to electronic excitations directly coupled to the elastic collision. The stability of the formvar film under electron radiation was studied. A reduction in thickness of the film with increasing fluence, as well as the preferential depletion of hydrogen, was found. Possible improvements of the experimental configuration for this type of experiments are discussed. (C) 2002 Elsevier Science B.V. All rights reserved.
\end{abstract}

PACS: $25.30 . \mathrm{Bf} ; 81.70 . \mathrm{Jb} ; 82.80 . \mathrm{Yc}$

Keywords: Hydrogen detection; Elastic scattering; Elastic electron scattering; Chemical composition analysis; Chemical depth and dopant profiling; Rutherford backscattering (RBS), and other methods of chemical analysis

\section{Introduction}

Electron microscopy in combination with elemental analysis is one of the major tools used to develop our knowledge in areas ranging from materials science to biology. Elemental analysis relies on the excitation of core electrons. In electron energy loss spectroscopy (EELS) edges in the measured loss distribution related to these core levels are used very effectively for the

E-mail address: maarten.vos@rsphysse.anu.edu.au (Maarten Vos)

http://wwwrsphysse.anu.edu.au/ampl/ems/ems.html. quantitative study of the composition [1]. Alternatively for thick samples, we can use the decay process of the core holes (by Auger electrons emission or X-ray emission) as the basis of the analysis of the surface composition. However, in all the three cases no unique signature of the presence of hydrogen is obtained although its presence may affect the detailed shape of the spectra obtained (see e.g. [2]). Direct detection of the ubiquitous hydrogen by an electron beam has been an elusive goal. Indeed hydrogen concentrations are usually obtained using nuclear methods [3]. In this paper, we present a method that allows for the direct detection of hydrogen (and possibly 
other light elements) when present in large concentrations. The experiment described uses a rather large-diameter electron beam $(0.1 \mathrm{~mm})$ and spatial resolution was not the main objective. I will discuss what kind of spatial resolution can be obtained, in principle, with this technique.

In many spectrometers, the energy analysis of elastically scattered electrons is often used to determine the experimental energy resolution: the convolution of the energy spread of the electron source and the resolving power of the electron analyser determines its width. However, the nomenclature 'elastic' is somewhat misleading. If an energetic electron with momentum with magnitude $k$ is deflected over an angle $\theta_{\mathrm{s}}$ by a (stationary) nucleus with mass $m$, its momentum changes by an amount $|q|$. Hence, the kinetic energy transferred to the nucleus is

$E=\frac{|q|^{2}}{2 m}=\frac{2}{m}\left(|k| \sin \left(\frac{1}{2} \theta_{\mathrm{s}}\right)\right)^{2}$.

In the case of impinging ions, the energy transfer is large and this makes Rutherford backscattering a powerful technique for the study of the composition of thin films. Compared to the mass of ions the small mass of an electron seems negligible (1836 times smaller than that of a proton). Hence, in electron-nucleon collisions, the mass of the target atoms is considered to be infinite and the energy transfer associated with an electron deflection is neglected. In the present paper we describe electron scattering experiments, in which 20-30 keV electrons are deflected over $44.3^{\circ}$. In these experiments collisions with extremely large momentum transfers, up to 35 a.u. are studied. (We use here atomic units (a.u.). One atomic unit of momentum corresponds to $1.89 \AA^{-1}$.) In comparison the momentum transfer in traditional EELS experiments is typically $<1$ a.u. [4]. For 35 a.u. momentum transfer to a proton the corresponding energy transfer is $q^{2} / 2 m$ i.e. $\simeq 0.33$ Hartree or $9.1 \mathrm{eV}$. This is an amount that is large compared to our experimental resolution $(0.6 \mathrm{eV})$. In the Rutherford backscattering literature, one expresses the energy loss due to the collision of the projectile ion with a target atom in terms of the kinematic factor. This kinematic factor is calculated from the target and projectile mass and the scattering angle [5]. That calculation is completely equivalent to the arguments given here, with the caveat that relativistic corrections become important for electrons at much lower energies than for ions. The assumption that $\gamma=\left(1+v^{2} / c^{2}\right)^{-1 / 2}=$ 1 , made implicitly in the familiar kinematic factor formula [5], results for the case of electrons in an error in the calculated energy loss of $10 \%$ at $25 \mathrm{keV}$. Indeed even at the modest energies of the present experiment, the agreement between experiment and theory improved noticeably when these relativistic corrections are taken into account.

\section{Experimental set-up}

The experiments were done in our (e, 2e) spectrometer. This is described in detail elsewhere [6]. Indeed the current experiment was triggered by the observation of an anomalous width of the elastic peak used in the calibration procedure of the $(\mathrm{e}, 2 \mathrm{e})$ analysers. Here, we briefly describe the essential parts as far as this paper is concerned. An outline is given in Fig. 1. An electron gun $(\mathrm{BaO}$ cathode, energy spread $0.3 \mathrm{eV}$ ) produces an electron beam of $600 \mathrm{eV}$. The sample is in an enclosure that floats at voltages between 19.4 and $29.4 \mathrm{keV}$. Thus, the electrons are accelerated up to 20-30 keV and impinge on a thin film (spot size $0.1 \mathrm{~mm}$ diameter, beam current typically $5 \mathrm{nA}$ ). Only electrons transmitted through this film and scattered over $44.3^{\circ}$ are detected by the electrostatic analyser. This angular selection is done by an $0.5 \mathrm{~mm}$ wide slit, $130 \mathrm{~mm}$ away from the sample. These slits are slightly curved in such a way that we detect over a $10^{\circ}$ sector of a $44.3^{\circ}$ cone [6]. After passing through these slits the electrons are retarded and focussed by a lens system. They enter the hemispherical analyser with a pass energy near $400 \mathrm{eV}$. Transmitted electrons are detected by a pair of channel plates followed by a positionsensitive detector (PSD, a resistive anode). The precise energy and azimuthal angle can be derived from the coordinates of the PSD.

In order to demonstrate this technique we used a thin formvar film. This polymer (nominal composition $\mathrm{C}_{8} \mathrm{O}_{2} \mathrm{H}_{14}$ ) should give us a high density of hydrogen and is most conveniently prepared as a 

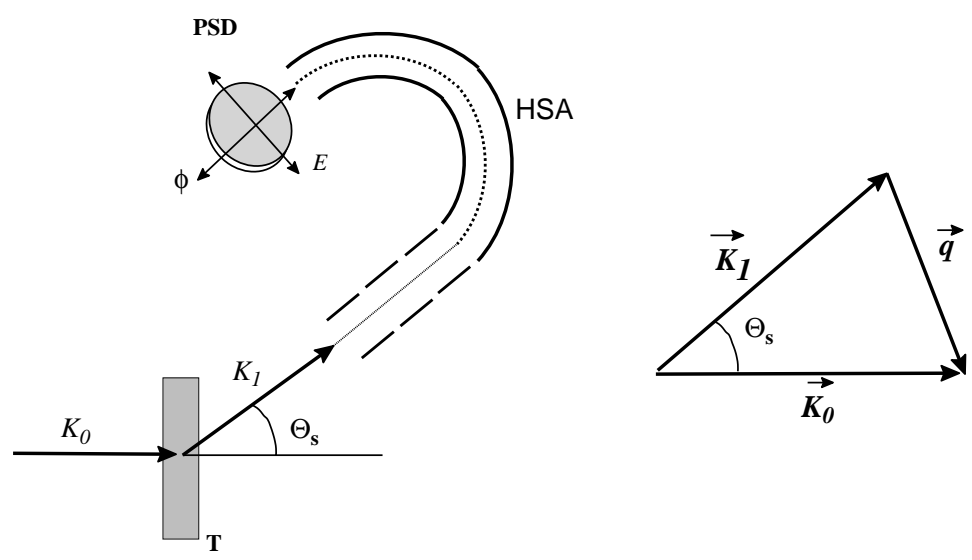

Fig. 1. The outline of the spectrometer (left). Electrons scattered over $\theta_{\mathrm{s}}$ are retarded by a lens system and analysed for energy in a hemispherical sector analyser (HSA) and detected using a position-sensitive detector (PSD). In the right panel we show the relation between the incoming $\left(\mathbf{k}_{0}\right)$, outgoing $\left(\mathbf{k}_{1}\right)$ and transferred momentum $(\mathbf{q})$.

free-standing film. In practise, we can collect a reasonable spectrum of these films (thickness approximately $20 \mathrm{~nm}$ ) in 2 min, i.e. using a dose of $0.006 \mathrm{C} / \mathrm{cm}^{2}$.

\section{Results}

The results of these measurements are shown in Fig. 2. Three different energies $E_{0}$ for the impinging electrons were used: 20, 25 and $30 \mathrm{keV}$. Electron gun and analyser potential were kept fixed, and only the high voltage applied to the target area was varied. The energy resolution depends on the stability and ripple of the gun and analyser potentials (which are all $<1 \mathrm{keV}$, stability and ripple better than $0.1 \mathrm{~V}$ ) but not on that of the sample high voltage (up to $30 \mathrm{keV}$ ). The analyser lens settings had to be adjusted so that for each energy the lenses focussed the incoming electrons at the entrance of the analyser. The main elastic peak is due to electrons scattered from carbon and oxygen. In these plots we choose, for convenience, the zero point of our energy loss scale to coincide with this peak position. However, even for carbon and oxygen we have an energy loss of $0.4-0.8 \mathrm{eV}$ under these conditions. The main peak has a width (full-width at half-maximum) close to $0.9 \mathrm{eV}$ in all the three cases indicating very similar level of focussing in the retardation lens system. A second

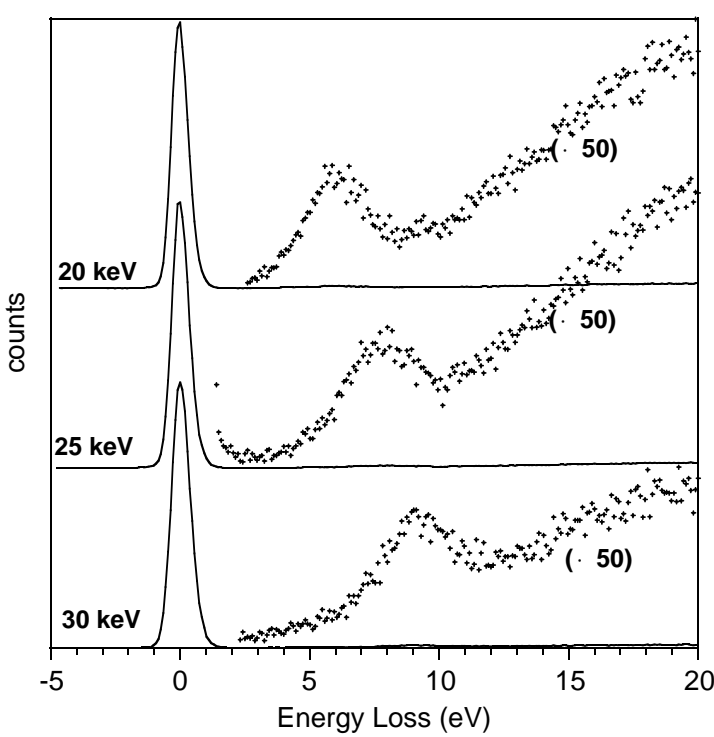

Fig. 2. The measured energy loss spectra for different energies. The intensity has been multiplied 50 times for energy loss exceeding $2 \mathrm{eV}$.

somewhat broader peak was seen at an energy loss position varying from 6 to $9 \mathrm{eV}$. It will be argued that this peak is due to electrons elastically scattered from hydrogen. Finally, there is a third, very broad structure peaking near $22 \mathrm{eV}$. As there are no inelastic scattering events that deflect an electron over $45^{\circ}$ and have an energy loss near 


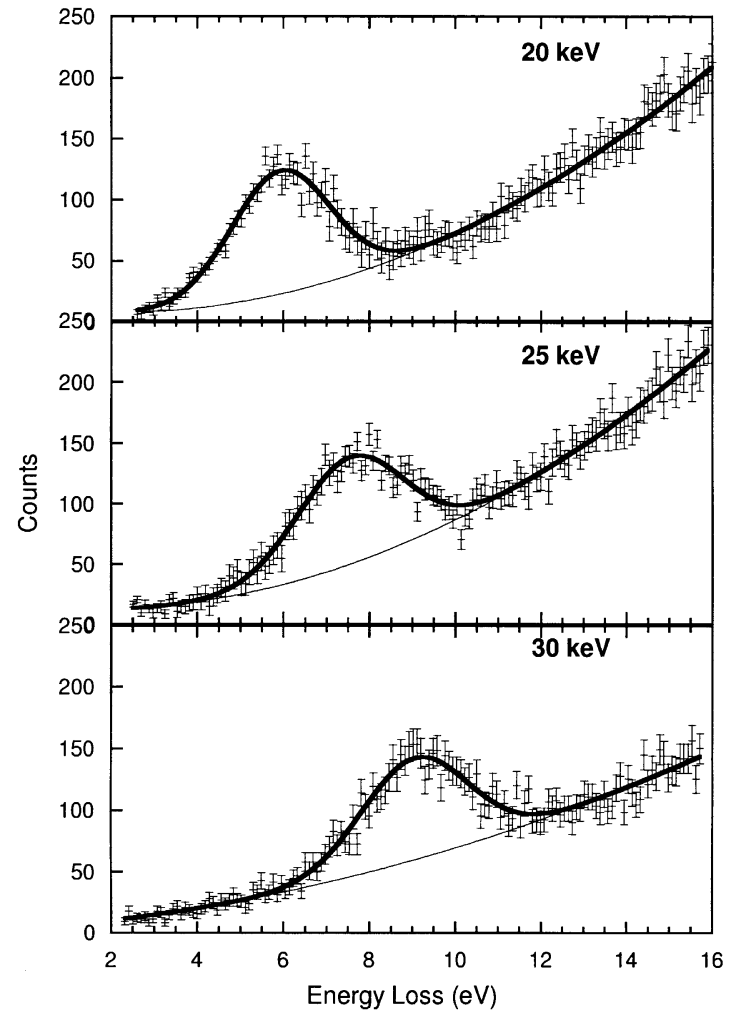

Fig. 3. The decomposition of the hydrogen part of the spectrum in terms of background and hydrogen elastic peak as determined from a least-squares fit.

$22 \mathrm{eV}$, we interpret this peak as being due to electrons that have experienced both a large-angle elastic deflection and an additional electronic excitation (plasmons and interband transitions). This part increases relative to the elastic peak with sample thickness. The thickness of the formvar is near one inelastic mean free path for $25 \mathrm{keV}$ electrons. The shape of this third structure does not vary with electron energy, only its intensity decreases with increasing energy, due to the gradual increase of the inelastic mean free path, and hence the decreased probability of multiple scattering.

In order to get a more quantitative description of the measurement we fitted the hydrogen part of the spectrum. The background was described by a quadratic polynomial, the peak itself by a Gaussian distribution. The results of these fits are shown in Fig. 3 and summarised in Table 1. The calculated shift $\Delta E_{\mathrm{obs}}$ is the difference between the energy loss for scattering of hydrogen and the mean energy loss for scattering of carbon and oxygen as the contribution of these atoms is not resolved. The observed shift between the main peak and the hydrogen peak $\Delta E_{\mathrm{obs}}$ agrees with the calculated shift $\Delta E_{\text {calc }}$ within $2 \%$. This validates the assignment of the small peak to electrons backscattered from hydrogen. Surprisingly, the width of the hydrogen peak $(\simeq 4 \mathrm{eV})$ is much larger than the width of the main peak $(\simeq 1.0 \mathrm{eV})$. This is due to vibrational motion of the hydrogen changing the kinematics of the collision. This effect opens the possibility of measuring the Compton profile of atomic vibrations and is discussed in a separate paper [7]. For analytical application the ratio of the intensity of the main line to the intensity of the hydrogen line is of more interest. In the case of Rutherford scattering the cross-section varies as $Z^{2}$ with $Z$ being the atomic number. Tabulated cross-sections for elastic electron scattering, based on the electron wave function, agree with this dependence for present conditions, indicating that screening of the nucleus by the target electrons is not important here [8]. Thus, based on a composition of $\mathrm{C}_{8} \mathrm{O}_{2} \mathrm{H}_{14}$ we would expect the intensity ratio of the hydrogen peak relative to the main peak (due to oxygen and carbon $)$ to be $14:\left(8 \times 6^{2}+2 \times 8^{2}\right) \simeq 1: 29.7$. Experimentally we find that the intensity ratio of

Table 1

A summary of the measured and calculated quantities as discussed in the text

\begin{tabular}{lllllll}
\hline $\begin{array}{l}E_{0} \\
(\mathrm{keV})\end{array}$ & $\begin{array}{l}q \\
(\text { a.u. })\end{array}$ & $\begin{array}{l}\Delta E_{\text {obs }} \\
(\mathrm{eV})\end{array}$ & $\begin{array}{l}\Delta E_{\text {calc }} \\
(\mathrm{eV})\end{array}$ & $\begin{array}{l}\text { FWHM } \\
(\mathrm{eV})\end{array}$ & $\begin{array}{l}I_{\mathrm{H}}: I_{\text {main }} \\
(\text { obs. })\end{array}$ & $\begin{array}{l}I_{\mathrm{H}}: I_{\text {main }} \\
(\text { calc. })\end{array}$ \\
\hline 20 & 29.2 & 5.9 & 5.8 & 2.6 & $1: 41.1$ & $1: 29.7$ \\
25 & 32.9 & 7.5 & 7.4 & 2.9 & $1: 44.8$ & $1: 29.7$ \\
30 & 35.9 & 9.0 & 8.8 & 2.9 & $1: 51.7$ & $1: 29.7$ \\
\hline
\end{tabular}


the main line to hydrogen line is between 1:40 and 1:50. Clearly the hydrogen peak is smaller than expected, and the discrepancy seems to increase with energy. This is currently not understood.

There are quite a few possible grounds for this discrepancy. Inadequacy of the fitting procedure, additional energy loss as part of the elastic collision with hydrogen and depletion of hydrogen due to the electron beam-induced decomposition of the films. We will discuss these possibilities briefly.

There are at least two critical assumptions made while fitting the experimental data: One assumption is that the background can be described by a polynomial of second order in energy, the other that the line shape can be described by a Gaussian function. The background obtained appears reasonable. A change of $50 \%$ in area cannot be easily obtained with a smooth varying background. Moreover, as the hydrogen contribution changes position with beam energy, the background reveals itself to be a rather smooth function of energy loss. Thus, it seems unlikely that the discrepancy originates in problems with the background substraction.

More difficult is the justification of the Gaussian line shape. Clearly, the hydrogen feature is much $(\simeq 4 \times)$ broader than the main elastic peak. Thus, the experimental contribution to the hydrogen line width is very small. As hinted before we think that the excess broadening is due to motion of the protons. However, until this is fully quantitatively understood we cannot justify the Gaussian line shape rigorously. For example, the assumption of a Lorentzian line shape would increase the area of the hydrogen feature significantly.

Another possibility is the unresolved presence of shake-like satellites associated with the hydrogen feature. The proton acquires up to $9 \mathrm{eV}$ kinetic energy in the collision. It is, thus rather unlikely that the chemical bond will survive this violent event. (The heat of formation of a $\mathrm{C}-\mathrm{H}$ bond is near $4 \mathrm{eV}$.) Excitation of the bonding electrons to excited states, in addition to the kinetic energy transfer may cause part of the hydrogen peak intensity to be shifted to higher loss values, due to these electronic excitations. It would be interesting to study these processes in the gas phase using, for example, ethane. Here, we can choose the density of the gas arbitrarily low, so that the multiple scattering background would vanish. Shake features would then be easily observable, and their intensity and position would provide insight in to the break-up of molecules after a well-defined perturbation.

Finally, it could be that the electron beam has changed the sample composition. In order to test this, and to underline that this method has practical applications, we studied the effect of irradiation on the sample composition. In the above all spectra were taken at fresh spots. Now we compare the first spectra with those obtained after irradiation of the sample with $0.35 \mathrm{C} / \mathrm{cm}^{2}$ $\left(E_{0}=25 \mathrm{keV}\right)$. The results are shown in Fig. 4 . The spectra are normalised in such a way that the main elastic peak have equal height. Clearly, the multiple scattering contribution has decreased somewhat due to the irradiation. However, there is still a well-defined hydrogen peak present. The ratio $I_{\mathrm{H}}: I_{\text {main }}$ has decreased by $22 \%$.

Radiation damage in organic compounds has been studied before by Egerton [9]. He used the ratio of elastic-to-inelastic scattering as a guide to estimate the amount of hydrogen removed. Parkinson et al. [10] studied the diffraction pattern of organic crystals and the amount of hydrogen seen in a mass spectrometer. These and other similar studies would clearly benefit from measurements such as the one described in this paper.

\section{Outlook and conclusion}

The current spectrometer was not designed for the measurements described here. Thus, it is more than likely that a spectrometer designed specifically with hydrogen detection in mind would perform better by at least an order of magnitude. The first obvious design parameter that could be varied is the scattering angle. The angle of $44.3^{\circ}$ was required for the (e, 2e) measurement. A truly backward angle seems more appropriate for the current experiment, as this would maximise the separation of hydrogen from heavier atoms and possibly resolve other light elements. However, a larger scattering angle would be at the expense of 

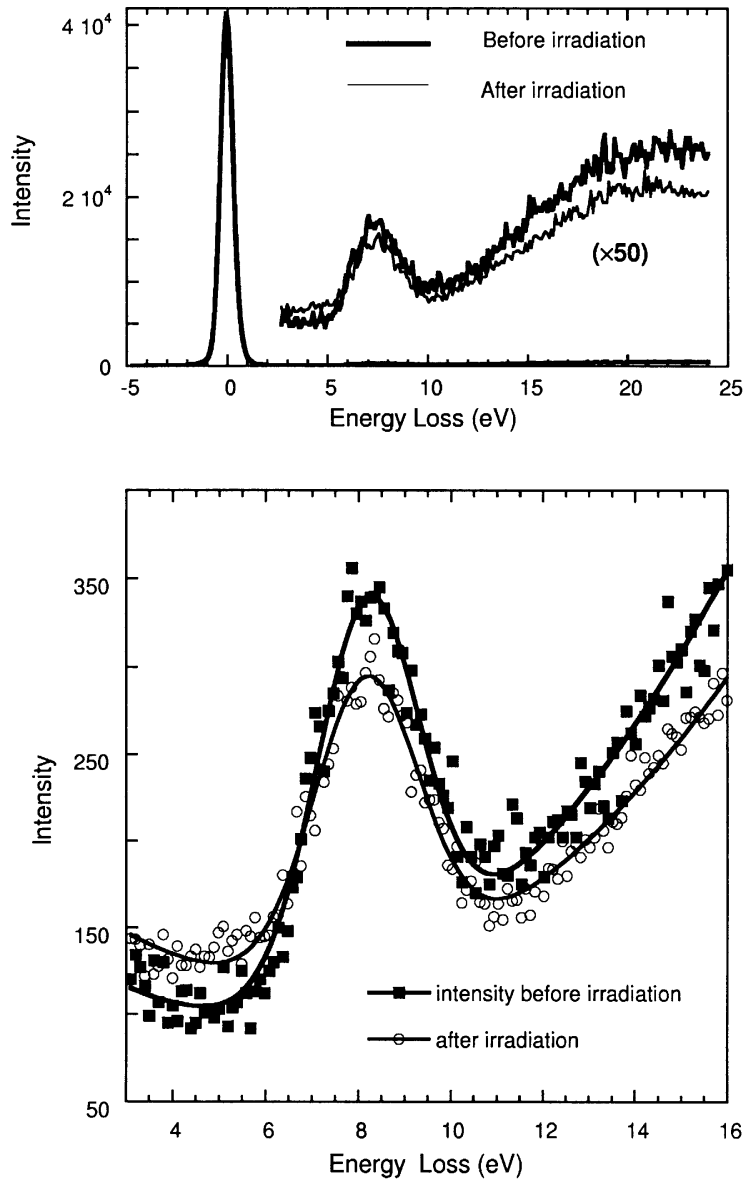

Fig. 4. The dependence of the measurement on electron irradiation. The top panel shows that the formvar film decreases in thickness during irradiation. The loss part that is due to multiple scattering (elastic scattering and inelastic scattering) decreases in intensity relative to the main peak (due to elastic scattering only). In the mean time the hydrogen peak decreases as well relative to the main peak, indicating preferential release of these atoms.

cross-section but it would make application to thick samples feasible, provided that the ratio of the background (due to multiple scattering) to the signal is sufficiently small.

The electron analyser should be designed in such a way that the opening angle is maximised. In the current experiment the width of the slits was dictated by the momentum resolution requirements of the (e, 2e) experiment, a consideration not relevant for hydrogen detection. Note that, if one increases the opening angle, aberrations in the retarding lens system tend to increase, reducing the obtained energy resolution. Another requirement is that the lens system should be able to focus over a wide energy range, which will allow the operator to chose the impinging electron energy and hence control the position of the hydrogen signal in such a way that the background contribution is most easily estimated.

By scanning a focussed electron beam one can, of course, get a map of the hydrogen distribution. The spatial resolution would be limited by the beam dose that can be used without significantly altering the sample. Note that improving the energy resolution will not improve performance significantly, as these features have a large intrinsic width. In the formvar case most of the hydrogen is still present after a radiation dose $50 \times$ that is required for a spectrum. Thus, with the current spectrometer these measurements are feasible with a change in beam diameter (i.e. spatial resolution) from 0.1 to $0.01 \mathrm{~mm}$. An optimisation of the electron optics could improve the detection efficiency at least by a factor of 10 , hence, allowing for smaller beams or more radiation damagesensitive substrates. Larger beam current would decrease the data acquisition time.

I would like to stress here a difference in description of energy loss processes in the (ion) Rutherford backscattering literature and electron spectroscopy. In the former, energy loss is described in terms of $\mathrm{eV} / \stackrel{\AA}{A}$. Ions lose energy for every atomic layer penetrated. There is a variation around the mean energy lost per layer, called straggling, and the straggling causes deterioration of the depth resolution with increasing depth. In electron spectroscopy one describes the energy loss in terms of inelastic events separated by trajectories (larger than the interatomic distance) without energy loss. The average length of these nonscattering distances are referred to as the inelastic mean free path (a few tens of $\mathrm{nm}$ at the current energy). The interpretation of these electronRutherford backscattering experiments is along the line of electron spectroscopy. Consequently, in a transmission experiment we have no depth resolution. In a reflection experiment (if possible) one would collect information from a surface layer with a thickness of roughly $0.5 \times$ mean free path. 
This depth could be varied by changing the energy $E_{0}$ or the angle of incidence.

In conclusion we have demonstrated a new technique, based on a electron beam, that directly monitors the hydrogen concentration in a thin film. In order to fully develop this technique for quantitative applications it will be necessary to understand why the intensity ratio of the main line and the hydrogen satellite is not correctly described by the Rutherford cross-section. The intensity of the signal is such that in an optimised design modest spatial resolution in the $1-10 \mu \mathrm{m}$ range should be obtainable.

\section{Acknowledgements}

I would like to thank Lou Chadderton and Bob McEachran for Erich Weigold for helpful discussions. The author is supported by a fellowship of the Australian Research Council.

\section{References}

[1] R. Egerton, Electron Energy-loss Spectroscopy in the Electron Microscope, Plenum Press, New York, 1986.
[2] R. Leapman, S. Sun, Cryo-electron energy loss spectroscopy: observations on vitrified hydrated specimens and radiation damage, Ultramicroscopy 59 (1995) 71-79.

[3] P. Khabibullaev, B. Skorodumov, Determination of hydrogen in materials, nuclear physics methods, in: Springer Tracts in Modern Physics, Vol. 117, Springer, Berlin, 1989.

[4] J. Fink, M. Knupfer, S. Atzkern, M. Golden, Electron correlation in solids, studied using electron energy-loss spectroscopy, J. Electron Spectrosc. Relat. Phenom. 117-118 (2001) 287.

[5] W. Chu, Backscattering Spectrometry, Academic Press, New York, 1978.

[6] M. Vos, G. Cornish, E. Weigold, A high-energy (e, 2e) spectrometer for the study of the spectral momentum density of materials, Rev. Sci. Instrum. 71 (2000) 3831.

[7] M. Vos, Observing atom motion by electron-atom compton scattering, Phys. Rev. A, 65 (2002) 012703.

[8] M. Riley, C. MacCallum, F. Briggs, Theoretical electronatom elastic scattering cross-sections, At. Data Nucl. Data Tables 15 (5) (1975) 443.

[9] R. Egerton, Measurement of the inelastic/elastic scattering ratio for fast electrons and its use in the study of radiation damage, Phys. Stat. Solidi A 37 (1976) 663.

[10] G. Parkinson, M. Goringe, W. Jones, W. Rees, J. Thomas, J. Williams, Electron induced damage in organic molecular crystals: some observations and theoretical considerations, in: J.A. Venables (Ed.), Development in Electron Microscopy and Analysis, Academic Press, New York, 1976, p. 316. 